\title{
Correlation of the Electrophysiological and Optical Coherence Tomography Changes in patients with Thyroid-Associated Ophthalmopathy
}

\section{Amany Abd El-Fattah El-Shazly ( $\sim$ amany_elshazly@ymail.com )}

Ain Shams University Faculty of Medicine https://orcid.org/0000-0002-4194-111X

\section{Mahmoud Ahmed Elsamkary}

Ain Shams University Faculty of Medicine

Tamer Abdel Fattah Badran

Assiut University Faculty of Medicine

\section{Randa Hesham Ali Abdelgawad}

Ain Shams University Faculty of Medicine

\section{Research article}

Keywords: Central foveal (CFT) thickness, Retinal nerve fiber layer (RNFL) thickness, Pattern electroretinogram (PERG), Pattern visual evoked potential (PVEP), Retinal Response Density (RRD), Thyroid-Associated Ophthalmopathy (TAO)

Posted Date: January 17th, 2020

DOI: https://doi.org/10.21203/rs.2.21158/v1

License: (c) (1) This work is licensed under a Creative Commons Attribution 4.0 International License. Read Full License 


\section{Abstract}

Purpose: to study the structural and functional changes of the optic nerve \& macula in patients with thyroid-associated ophthalmopathy (TAO) patients.

Methods: Cross-sectional clinical study including 40 cases with TAO and 40 age and sex-matched healthy participants as a control. Complete ophthalmological assessment, evaluation of the proptosis, spectral domain OCT, and electrophysiological investigations (pattern electroretinogram [PERG], multifocal ERG (mfERG) \& visual evoked potentials (PVEP) were performed to all participants.

Results: Retinal nerve fibre (RNFL), central foveal (CFT) thickness and mean inner macular ring thickness are thinner in cases with proptosis. MfERG showed lower Retinal Response Density1 (RRD1), Ring 1 P1 amplitude and lower five-rings $\mathrm{N} 1$ amplitude. Central foveal thickness showed significant positive correlation with VA, BCVA, P50 amplitude and R1 N1 amplitude $(r=0.64,0.65,0.40$ and 0.51 with $p<0$ $.001,<0.001,<0.001$ and $<0.001$ respectively), and negative correlation with duration of the disease, degree of proptosis, clinical activity score and R1 N1 latency $(r=-0.59,-0.78,-0.41 \&-0.90$ with $p<0.001$, $<0.001,<0.001 \&<0.001$ respectively). RNFL thickness showed negative correlation with duration of the disease, degree of proptosis and clinical activity score $(-0.77,-0.71 \&-0.85$ with $p<0.001,<0.001 \&<0$ .001 respectively). Multiple regression analyses showed that the degree of proptosis and P50 amplitude were the most important determinants for CFT ( $p=0.03 \& 0.02)$; whereas the duration of the disease, and activity score were the most important determinants for average RNFL thickness $(p=0.004$, and $<0.001$ respectively).

Conclusion: In the absence of fundus changes, macular thinning together with functional changes detected by PERG and mfERG could be used as good predictors of subclinical retinopathy in the cases of TAO.

\section{Introduction}

Thyroid-associated ophthalmopathy (TAO) is autoimmune inflammation of the orbital tissues. It is associated with inflammatory cellular infiltration by lymphocytes, plasma cells, macrophages, and mast cells to the orbital fat, interstitial tissues, and lacrimal glands, accompanied by retention of fluid and accumulation of glycosaminoglycans. This induces increase in the volume of orbital contents and secondary increase of intraorbital pressure. $(1,2)$ Elevation of intraorbital pressure and the extraocular muscles swelling at the apex lead to compressive optic neuropathy and loss of vision. (3)

It was apparent that affection of optic disc function may be present in TAO patients without noticeable extraocular muscle swelling. (4-6) So, some diagnostic and follow-up tests were previously used to assess the visual function of the patients with TAO, such as, contrast sensitivity test, visual field test, visual evoked potential, and color vision test. Optical coherence tomography (OCT) is an alternative and non-invasive test to assess the structure of the macula and the optic disc. Previously, only three studies studied retinal nerve fiber layer thickness and optic disc morphology, measured by OCT in TAO patients. 
(7-9) In the patients with TAO the thicknesses of macula and inferior RNFL were thinner when compared with healthy controls. (10)

Dysthyroid optic neuropathy (DON) is the commonest reason of visual loss in thyroid associated orbitopathy (TAO). (11)

Electrophysiological studies, including visual evoked potential (VEP) and electroretinogram (ERG) were adopted to provide objective evaluation and correlation with the presence and/or severity of DON. (12)

The pattern reversal ERG (PERG) and the photopic negative response (PhNR) of the full-field ERG provide objective measures of retinal ganglion cell function. (13)

The patient presented with diminution of vision even those with mild proptosis, so the aim of our study is to assess the structural and functional changes of the optic nerve \& macula in patients with thyroidassociated ophthalmopathy (TAO) to explain the diminution of vision.

\section{Subjects And Methods}

Our study was a prospective cross-sectional study, involving 40 patients with proptosis due to hyperthyroidism and 40 healthy adults as control. It was conducted in Ophthalmology department Ain Shams University between March 2018 and March 2019. The age range was between 25 and 72 years old, with the controls age and sex matched.

We excluded patients with pathological myopia or with any retinal disorder such as diabetic retinopathy. Patients suffering from uveitis, drusen, glaucoma, previous eye trauma, retinal vascular abnormalities, age-related macular degeneration, opaque media, previous optic nerve disease or other chronic eye diseases were excluded. Patients with history of intraocular surgery, refractive surgery, intravitreal injection, or systemic diseases other than thyroid disease were excluded as well.

We started our study by taking a good history from the patients. The diagnosis of hyperthyroidism was established by testing the serum level of TSH, free T3 and T4. All subjects underwent full ophthalmological examination, including best-corrected visual acuity (BCVA), slit-lamp examination, Goldman applanation tonometry, indirect ophthalmoscopy, ocular motility examination, evaluation of the proptosis using Hertel's exophthalmometer. We used initial clinical activity score (CAS) which includes only first seven criteria. The items included the following: (1) spontaneous orbital pain, (2) gaze-evoked orbital pain, (3) eyelid swelling that is considered to be due to active (inflammatory phase) TAO, (4) eyelid erythema, (5) conjunctival redness that is considered to be due to active (inflammatory phase) TAO, 6) chemosis, and (7) caruncle inflammation or plica. CAS $\geq 3 / 7$ indicated active TAO. (14)

Our procedure was performing Spectral Domain Optical Coherence Tomography (SD-OCT) (retinascan RS-3000 advance; NIDEK, Gamagori, Japan) and mfERG stimulation (Roland Consult RETI port/scan 21; Roland Consult, Brandenburg, Germany). 


\section{Axial Length Measurement}

The AL was measured using A-scan ultrasonography (PacScan 300A). The cornea was anesthetized with one drop of topical $0.4 \%$ benoxinate hydrochloride ophthalmic solution (ALTAIRE Pharm, Inc). For each eye, five readings were obtained to calculate an average value. The standard deviation was $0.1 \mathrm{~mm}$ for each eye. Eyes with axial length more than $25 \mathrm{~mm}$ were excluded.

\section{Central Corneal Thickness Measurement}

Measurement of CCT was conducted using SDOCT (retinascan RS-3000 advance) by means of the anterior segment optical coherence tomography (OCT) program, where we used the anterior segment adaptor (a special lens mounted on the camera device to capture the anterior segment and the cornea). We used the single corneal line scan horizontally with the scan width of $6 \mathrm{~mm}$ centered on the centre of the cornea in which the single scan was averaged from $10 \mathrm{~A}$-scans to get the best quality image and reduce noise. The resolution of the single scan of the $10 \mathrm{~A}$-scans was 1,024 points. Then, the CCT was measured from the superficial hyperreflective line representing the corneal epithelium to the deepest hyper-reflective line representing the corneal endothelium.

\section{Pattern Visual-Evoked Potentials Recording}

The PVEP test parameters were in accordance with the International Society for Clinical Electrophysiology of Vision (ISCEV) standard. Pattern visual evoked potential was recorded with RETI-port/scan 21 (Roland Consult, Brandenburg, Germany). Pupils were not dilated. Stimulation was monocular. Eyes were refracted for the stimulus viewing distance (one meter). Parameters of the stimulation were as follows: 2199 cathode ray tube monitor with a frame rate of 75 frames per second (fps), black/white reversing checkerboard was presented to the patient with a check size of $1^{\circ} 49$ and $0^{\circ} 169$, Michelson contrast was $97 \%$, temporal frequency of 2 reversals per second (rps) $(1 \mathrm{~Hz})$, luminance for the white elements was 120 candelas per square meter $(\mathrm{cd} / \mathrm{m} 2)$ (mean luminance: $55 \mathrm{~cd} / \mathrm{m} 2)$. According to the international $10 / 20$ system, the scalp electrodes were placed relative to bony landmarks, in proportion to the size of the head.

The active-gold disk electrode was placed on the scalp over the visual cortex at $\mathrm{Oz}$, the reference-gold disk electrode at Fz, while ground-gold disk electrode was placed on the forehead. Central fixation was maintained and properly monitored. Parameters of the recording system were as follows: filters of frequency $1 \mathrm{~Hz}$ to $100 \mathrm{~Hz}$ (notch filters were switched off), artifact reject threshold was $50 \mathrm{mV}$, and sweep time of 300 milliseconds (ms), average of 100 sweeps. Two consecutive waveforms were recorded, then averaged off-line and analysed. The amplitude and time of the P100-wave with manual correction to the automatic cursor placement were also parsed. To evaluate the bioelectrical function of the macular versus more peripheral regions of the retina, stimulation with the small $\left(0^{\circ} 169\right)$ and large $\left(1^{\circ} 49\right)$ check stimuli were measured. (15)

\section{Pattern Electroretinogram Recording}


The PERG was recorded with the RETI-port/scan 21 using a protocol implemented in the original software of the system. Monocular stimulation was used, together with appropriate refractive error correction in relation to the eye-screen distance. Examination was interrupted when frequent blinking or fixation losses were observed (the patient was monitored with a TV camera). The patients' pupils were not dilated, and central fixation was ascertained. Parameters of the PERG stimulation were as follows: 2199 cathode ray tube monitor with a frame rate equal to $75 \mathrm{fps}$ was used; black and white reversing checkerboard $\left(30^{\circ}\right.$ field of view "FOV") was presented to the patient with a check size equal to $1^{\circ} 29$; temporal frequency of 4.6 reversals/second (rps) $(2.3 \mathrm{~Hz}$ ); Michelson contrast of $97 \%$; luminance for white elements was 120 $\mathrm{cd} / \mathrm{m} 2$. Electrodes used are as follows: ground-gold disk electrode was placed on the forehead, HawlinaKonec (HK)-loop electrode was hooked into lower fornix, and separate surface reference electrodes were placed on the skin near the ipsilateral outer canthus of each eye. Parameters of the recording system were as follows: amplifiers sensitivity of 20 microvolts $(\mathrm{mV}) /$ division filters, filters of frequency $1 \mathrm{~Hz}$ to $100 \mathrm{~Hz}$, artifact reject threshold was $95 \%$ (for the amplifiers range $\pm 100 \mathrm{mV}$ ), notch filters were off, and averaging of 200 sweeps, sweep time was 250 milliseconds (timebase: 25 ms/division). Two consecutive waveforms were recorded; then, they were off-line averaged and analysed. The PERG test parameters were in accordance with the International Society for Clinical Electrophysiology of Vision standard. (15)

\section{Multifocal electroretinogram (mfERG) procedure}

It was performed with the RETI-port/scan 21. The patient was prepared by pupil dilatation using $0.5 \%$ tropicamide. Golden cup electrodes were placed in the forehead as a ground electrode and in the outer canthi as reference electrodes. HK Loop electrodes were hooked into lower fornices as active electrodes. Acquisition of records was done monocularly.

The test was performed according to the guidelines of the International Society of Electrophysiology of Vision (ISCEV). In the normal room light (photopic condition), patients were asked to maintain fixation on a red fixation target at the center of stimulus matrix. We performed our mfERG using 61 scaled hexagons displayed on a cathode ray tube (CRT) monitor as the visual stimulus. Hexagon sizes were scaled with eccentricity to elicit approximately equal amplitude responses at all locations. The hexagons alternate between black and white according to pseudorandom binary m-Sequence, with luminance of $100 \mathrm{~cd} / \mathrm{m}^{2}$ in white hexagons and $2 \mathrm{~cd} / \mathrm{m}^{2}$ in black hexagons.

We performed two full mfERG recording sessions for each subject to take an average which is displayed in the form of five concentric rings. Ring (1) is the most central hexagon with radius of about $0.5 \mathrm{~mm}$ corresponding to the fovea $\left(0^{\circ}-2^{\circ}\right)$, ring 2 to the parafovea $\left(2^{\circ}-7^{\circ}\right)$, ring 3 to the perifovea $\left(7^{\circ}-13^{\circ}\right)$, ring 4 to the near periphery $\left(13^{\circ}-22^{\circ}\right)$, and ring 5 to the central part of the middle periphery $\left(22^{\circ}-30.5^{\circ}\right)$. (16)

\section{OCT methodology}

Optical coherence tomography OCT was performed with the Spectral Domain Optical Coherence Tomography (SD-OCT) (retinascan RS-3000 advance; NIDEK, Gamagori, Japan). We performed retinal thickness, choroidal thickness and nerve fiber layer thickness. 


\section{Macular Thickness}

Preparation for the procedure was done by mydriasis using tropicamide $0.5 \%$ eye drops. Our results were displayed by macular map composed of nine sectorial thickness measurements in three concentric circles with diameters of 1,3 and $6 \mathrm{~mm}$. The area bounded by the outer $(6 \mathrm{~mm})$ and middle $(3 \mathrm{~mm})$ circles forms the outer ring (OR) while the area bounded by the middle $(3 \mathrm{~mm})$ and inner $(1 \mathrm{~mm})$ circles forms the inner ring (IR). Each ring is divided into superior, nasal, inferior and temporal quadrants. SIM, IIM, NIM, TIM stand for superior of inner macula, inferior of inner macula, nasal of inner macula, and temporal of inner macula, respectively. SOM, IOM, NOM, TOM stand for superior of outer macula, inferior of outer macula, nasal of outer macula, and temporal of outer macula, respectively [16].

\section{OCT retinal nerve fiber layer (RNFL)}

The RNFL thickness was measured using the Disc Map Protocol $(6 \times 6 \mathrm{~mm})$. Aging effect on RNFL was taken into consideration according to the normative data base of our machine. Results were displayed in the form of 4 sectors superior, nasal, inferior and temporal. Cup/disc ratio was estimated. Our results were plotted in a curvilinear line that divide the results into normal, border line or abnormal. Each of the previously described procedures was performed by the same experienced operator for all the patients [15].

\section{Statistical Analysis.}

We analysed data using the Statistica software, version 12. Quantitative variables were expressed as mean \pm SD. Descriptive statistics were done using chi-square analysis. An independent sample t-test and Pearson's correlation analysis were used for the statistical analysis of the data. Regression analyses were done to assess the different factors that can affect the RNFL and macular thickness. Significance was evaluated at $p<0.05$ levels.

\section{Results}

\section{Table 1: Demographics in controls and patients with TAO:}

There were no statistically significant differences between the two groups regarding age, spherical equivalent, intraocular pressure, AL, and CCT. Although there is statistical difference between the two groups regarding VA and BCVA. Table 1

Fundus examination showed no abnormalities in the studied groups.

The degree of proptosis in the studied cases has a mean of $21.25 \pm 3.04 \mathrm{~mm}$ (range, 18-28). The mean disease duration was $1.63 \pm 0.70$ years (range, $1-3$ years) and the clinical severity score was $3.50 \pm 1.13$ (range, 2-5). Table 1 
The gender of participants was not statistically different in the two studied groups including control group (male/female ratio $28 / 12$ and cases with proptosis (male/female ratio $30 / 10$ ) with $(\chi 2=0.25$ and $p=0.62)$.

Table 2: Optical coherence tomographic measurements of retinal nerve fibre layer (RNFL) thickness in the studied groups:

Regarding retinal nerve fibre layer, there were significant differences among the two studied groups in the different areas. The retinal nerve fibre layer was significantly thinner in cases with proptosis than that of the control group. (Table 2)

Table 3: Optical coherence tomographic measurements of macular retinal thickness of the controls and patients with TAO:

The CFT and mean inner macular ring thickness are significantly lower in cases with proptosis $(P=0.002$ and $<0.001$ ) whereas mean outer macular thickness showed insignificant differences between the two groups. (Table 3)

Table 4. Pattern Electroretinogram (PERG) and Pattern visual evoked potential (PVEP) Parameters in the Studied Groups:

All Parameters of PERG and PVEP showed insignificant differences between the two groups; except for P50 amplitude which is statistically diminished than that of control (with $p=0.02$ ). Table 4

Table 5: Multifocal electroretinographic and statistical findings: five-rings retinal response density (RRD) of the controls and Patients with TAO:

RRD: Ring response density

$\mathrm{nV} / \mathrm{deg}^{2}$ : nanovolt per degree square.

Cases with proptosis had significantly lower RRD of R1 $(P=0.03)$ while showing insignificant differences between the two groups regarding R2, R3, R4, R5 response density. Table 5

Table 6: Multifocal Electroretinographic and Statistical Findings: Five- rings P1 amplitude and latency of the controls and patients with TAO:

Cases with proptosis had significantly lower ring1 $P 1$ amplitude $(P=0.02)$ while showing insignificant differences between the two groups regarding R2, R3, R4, and R5 P1 amplitudes. Whereas no statistical difference was found between the two groups regarding the five rings P1 latency. Table 6

Table 7: Multifocal Electroretinographic and Statistical Findings: Five- rings N1 amplitude and latency of the controls and patients with TAO:

Cases with proptosis had significantly lower five- rings N1 amplitude. Whereas no statistical difference was found between the two groups regarding the five rings N1 latency. Table 7 
Cases with proptosis had significantly lower four-quadrant N1 amplitude whereas there was no statistical difference between the two studied groups regarding the mean quadrant N1 Amplitude $(p=0.90)$. Also, no statistical difference was found between the two groups regarding the four-quadrant $\mathrm{N} 1$ latency except for the mean quadrant N1 latency which showed statistically significant difference between the 2 groups with ( $p$ value $=0.02)$. Table 7

\section{Figure 1. Correlation between R1 N1 amplitude and degree of proptosis.}

\section{Figure 2. Correlation between R1 N1 amplitude and clinical activity score.}

In cases with proptosis, central foveal thickness showed no significant correlation between age, spherical equivalent, $A L, C C T$, RRD1, ring1 P1 amplitude and ring1 P1 latency $(r=-0.17,0.03,-0.12,-0.24,0.18$, $-0.07,0.22$ and 0.05 respectively $\& p=0.29,0.85,0.46,0.14,0.26,0.67,0.17$ and 0.76 respectively); but showed significant positive correlation with VA, BCVA, P50 amplitude and R1 N1 amplitude $(r=0.64,0.65$, 0.40 and 0.51 with $p<0.0001,<0.0001,<0.0001$ and $<0.0001$ respectively), and negative correlation with duration of the disease, degree of proptosis, clinical activity score and R1 N1 latency $(r=-0.59,-0.78$, $-0.41 \&-0.90$ with $p<0.0001,<0.0001,<0.0001 \&<0.0001$ respectively). Figure $1 \& 2$

\section{Figure 3. Correlation between Average RNFL thickness and clinical activity score}

\section{Figure 4. Correlation between Average RNFL thickness and degree of proptosis.}

In cases with proptosis, RNFL thickness showed no significant correlation between age, spherical equivalent, $\mathrm{AL}, \mathrm{CCT}, \mathrm{VA}, \mathrm{BCVA}$, IOP, mean quadrant response density, mean quadrant $\mathrm{P} 1$ amplitude and mean quadrant $P 1$ Latency $(r=-0.07,0.08,0.10,-0.14,-0.05,-0.11,0.20,0.27,0.07 \& 0.15$ with $p=0.67$, $0.62,0.54,0.39,0.76,0.50,0.22,0.09,0.67 \& 0.36)$; but showed significant positive correlation with mean quadrant $\mathrm{N} 1$ amplitude $(r=0.48$ with $p<0.0001)$, and negative correlation with duration of the disease, degree of proptosis, clinical activity score and mean quadrant N1 latency $(r=-0.71 \&-0.77$ with $p<0$ $.0001 \&<0.0001$ respectively). Figure $3 \& 4$

\section{Table (8): Multiple regression analysis of different factors affecting CFT in in the patients with TAO:}

We plotted multiple regression analyses for patients with proptosis to show which factor in the study was the most important determinant of CFT. We found that CFT was not attributable to age, VA, BCVA, SE, AL, IOP, CCT, RRD1, duration of disease, ring1 P1 amplitude, ring1 P1 latency, ring1 N1 amplitude, ring1 N1 latency or clinical activity score, while degree of proptosis and P50 amplitude were the most important determinant for CFT ( $\beta=-0.56 \& 0.40$ and $p=0.03 \& 0.009$ ). Table 8

Table (9): Multiple regression analysis of different factors affecting RNFL thickness in in the patients with TAO:

Also, average RNFL thickness was not attributable to age, VA, BCVA, SE, AL, IOP, CCT, degree of proptosis; while duration of the disease, and clinical activity score were the most important determinant 
for average RNFL thickness ( $\beta=0.67$ and -0.81 respectively) ( $p=0.004$, and $<0.001$ respectively). Table 9

\section{Discussion}

We conducted this study to evaluate possible benefits of using electrophysiological investigations to detect retinal and visual pathway changes occurring in patients with TAO and to correlate them with the structural changes. We found that the average RNFL thickness and different RNFL (superior, inferior, nasal, and temporal quadrants) thicknesses were thinner in patients with proptosis. This is in agreement with Sayın et al. (17) who found that the retinal nerve fiber layer thicknesses were thinner in the patients with thyroid-associated ophthalmopathy. Forte et al. ${ }^{8}$ found that there was a decrease in RNFL in patients with Graves' ophthalmopathy when compared with the control group.

This is in contradiction with Romano et al. (18) who found that the average RNFL thickness was lower in the optic nerve compression group than in the control group, and the difference was not statistically different. Blum Meirovitch et al. (19) found that there is increased RNFL thickening. Neudorfer et al. (20) also, stated that there is an increased peripapillary RNFL thickness in patients with TAO and this increase in thickness could be attributed to initial RNFL edema caused by optic nerve compression.

Also, the CFT and mean inner macular ring thickness are significantly thinner in cases with proptosis; whereas mean outer macular thickness showed insignificant differences between the two groups. This is in agreement with Blum Meirovitch et al. (19) who found that eyes of TAO patients had a significant thinning of the inner macula compared to the eyes of the healthy control group and with Sayın et al. (17) who found also that the macular thickness was thinner in the patients with thyroid-associated ophthalmopathy in comparison to their controls

The macular thinning in our TAO eyes may be secondary to the mechanical compression on the retina by orbital contents or as a predominant feature in patients with autoimmune retinopathy due to damage caused by anti-retinal antibodies. (21) Another possible mechanism for macular thinning may be the decrease in blood flow to the retina of TAO patients. Fernandez-Buenaga et al. (22) found macular thinning in the eyes of patients with non-arteritic anterior ischemic optic neuropathy (NAION), which they attributed to the ischemic damage in the maculopapillary bundle that occurs in NAION. The decrease in blood flow in TAO disease may contribute to the macular thinning via vein congestion or arterial stenosis.

Some studies have addressed the impact of proptosis on various electrophysiologic studies. VEP was used in 14 studies (23-26) three studies tested PERG. (27-29) No study was found on flash or multifocal ERG. (11)

To the best of our knowledge, our study is the first to evaluate multifocal ERG changes in patients with proptosis due to TAO and to correlate these changes to that of the OCT.

In our recruited cases with proptosis, we found affection of visual acuities in patients compared with controls, and also no detectable retinal abnormalities by fundus examination, whereas mfERG response 
densities (RRD) showed significantly decreased value of ring1 in cases than in controls. Moreover, P50 wave of PERG showed statistically significant lower amplitude in the cases. This denotes that these patients are at an early stage of macular insult. Also, we noticed that there is lower four-quadrant N1 amplitude whereas there was no statistical difference between the two studied groups regarding the mean quadrant $\mathrm{N} 1$ amplitude. Worthy of notice is that electrophysiologic tests that showed significant differences among patients and controls were mfERG and P50 wave of PERG, which are the tests evaluating macular function. On the contrary, PVEP and N95 wave of PERG that assess visual pathway and optic nerve functions were statistically insignificant among the studied groups. These results might denote that TAO could affect the retina and macular area even without optic nerve insult.

We could not find any studies that address those changes in TAO before but Abazari et al. (21) correlated retinal atrophy and reduced macular thickness on OCT with corresponded depressed central response on multifocal ERG (mfERG) testing. He stated that those changes are predominant features in patients with autoimmune retinopathy. This may offer clues toward understanding the mechanism of visual dysfunction and the diagnosis of autoimmune retinopathy.

Blum Meirovitch et al. (19) found that the OCT findings suggest that the retina is involved in TAO, probably as early as the subclinical stage and the diagnosis of retinal changes earlier could prevent severe visual sequelae.

Moreover, we found that central foveal thickness showed significant positive correlation with VA, BCVA, P50 amplitude and R1 N1 amplitude, and negative correlation with duration of the disease, degree of proptosis, clinical activity score and R1 N1 latency.

Another explanation that the foveal and retinal function affection in our TAO eyes may be attributed to long-lasting chronic impairment of blood supply of the eyeball. (4)

Also, the RNFL thickness showed significant negative correlation with duration of the disease, degree of proptosis and clinical activity score.

We plotted multiple regression analyses for patients with proptosis to show which factor in the study was the most important determinant of CFT. We found that CFT was not attributable to age, VA, BCVA, SE, AL, IOP, CCT, RRD1, duration of disease, ring1 P1 amplitude, ring1 P1 latency, ring1 N1 amplitude, ring1 N1 latency or clinical activity score, while degree of proptosis was the most important determinant for CFT.

Also, average RNFL thickness was not attributable to age, VA, BCVA, SE, AL, IOP, CCT, degree of proptosis; while the duration of the disease and the clinical activity score were the most important determinants for average RNFL thickness.

Because TAO has an immunologic base, we believe there is a need for further studies to evaluate the presence of autoimmune retinopathy and correlate it with anti-retinal antibodies in TAO patients. 
Our study has several limitations, the main one is the small sample size of patients, although our results reached a level of significance, multicenter studies may be of more value, and cross-sectional design would not provide further and detailed results. Further studies are required to validate our findings and arrive at conclusions regarding the contribution of OCT and its correlation with functional changes detected with $\mathrm{mfERG}$ at the different stages of TAO.

\section{Conclusion}

In the absence of fundus changes, RNFL and macular thinning together with functional changes detected with PERG and mfERG could be used as good predictors for subclinical autoimmune retinopathy in the cases of TAO.

\section{References}

1. Menconi F1, Marcocci C2, Marinò M2. Diagnosis and classification of Graves' disease. Autoimmun Rev. 2014 Apr-May;13(4-5):398-402. doi: 10.1016/j.autrev.2014.01.013. Epub 2014 Jan 12.

2. Smith TJ1.Insights into the role of fibroblasts in human autoimmune diseases. Clin Exp Immunol. 2005 Sep;141(3):388-97.

3. Anderson RL1, Tweeten JP, Patrinely JR, Garland PE, Thiese SM. Dysthyroid optic neuropathy without extraocular muscle involvement. Ophthalmic Surg. 1989 Aug;20(8):568-74.

4. Beden Ü1, Kaya S2, Yeter V3, Erkan D4. Contrast sensitivity of thyroid associated ophthalmopathy patients without obvious optic neuropathy. ScientificWorldJournal. 2013 Dec 24; 2013:943789. doi: 10.1155/2013/943789. eCollection 2013.

5. Hallin ES1, Feldon SE, Luttrell J. Graves' ophthalmopathy: III. Effect of transantral orbital decompression on optic neuropathy. Br J Ophthalmol. 1988 Sep;72(9):683-7.

6. Kennerdell JS, Rosenbaum AE, El-Hoshy MH. Apical optic nerve compression of dysthyroid optic neuropathy on computed tomography. Arch Ophthalmol. 1981 May;99(5):807-9.

7. Wei YH1, Chi MC, Liao SL Predictability of visual function and nerve fiber layer thickness by crosssectional areas of extraocular muscles in graves ophthalmopathy. Am J Ophthalmol. 2011 May;151(5):901-906.e1. doi: 10.1016/j.ajo.2010.11.001. Epub 2011 Feb 18.

8. Forte R1, Bonavolontà P, Vassallo P. Evaluation of retinal nerve fiber layer with optic nerve tracking optical coherence tomography in thyroid-associated orbitopathy. Ophthalmologica. 2010;224(2):11621. doi: 10.1159/000235925. Epub 2009 Sep 2.

9. Sen E1, Berker D, Elgin U, Tutuncu Y, Ozturk F, Guler S. Comparison of optic disc topography in the cases with graves' disease and healthy controls. J Glaucoma. 2012 Dec;21(9):586-9. doi: 10.1097/IJG.0b013e31822e8c4f.

10. Sayın O, Yeter V, Arıtürk N. Optic Disc, Macula, and Retinal Nerve Fiber Layer Measurements Obtained by OCT in Thyroid-Associated Ophthalmopathy. J Ophthalmol. 2016; 2016:9452687. doi: 10.1155/2016/9452687. Epub 2016 Jul 17. 
11. lao TWU1, Rong SS1,2, Ling AN1, Brelén ME1,3, Young AL1,3, Chong KKL4,5. Electrophysiological Studies in Thyroid Associated Orbitopathy: A Systematic Review. Sci Rep. 2017 Sep 21;7(1):12108. doi: 10.1038/s41598-017-11998-0.

12. Wilsey LJ1, Fortune B. Electroretinography in glaucoma diagnosis. Curr Opin Ophthalmol. 2016 Mar;27(2):118-24. doi: 10.1097/ICU.0000000000000241.

13. Subekti I1, Boedisantoso A, Moeloek ND, Waspadji S, Mansyur M. Association of TSH receptor antibody, thyroid stimulating antibody, and thyroid blocking antibody with clinical activity score and degree of severity of Graves ophthalmopathy. Acta Med Indones. 2012 Apr;44(2):114-21.

14. Bartalena L, Baldeschi L, Dickinson A, Eckstein A, Kendall-Taylor P, Marcocci $C$, et al. Consensus statement of the European Group on Graves' orbitopathy (EUGOGO) on management of GO. Eur J Endocrinol 2008; 158:273-285.

15. El-Shazly AAE1, Farweez YA1, Hamdi MM1, El-Sherbiny NE1.Pattern Visual Evoked Potential, Pattern Electroretinogram, and Retinal Nerve Fiber Layer Thickness in Patients with Migraine during and after Aura. Curr Eye Res. 2017 Sep;42(9):1327-1332. doi: 10.1080/02713683.2017.1319490. Epub 2017 Jun 21.

16. El-Shazly AA1, Ebeid WM, Elkitkat RS, Deghedy MR. ELECTRORETINOGRAPHIC AND VISUAL-EVOKED POTENTIAL CHANGES IN RELATION TO CHELATION MODALITY IN CHILDREN WITH THALASSEMIA. Retina. 2017 Jun;37(6):1168-1175. doi: 10.1097/IAE.0000000000001315.

17. Sayın 01, Yeter V2, Arıtürk N3. Optic Disc, Macula, and Retinal Nerve Fiber Layer Measurements Obtained by OCT in Thyroid-Associated Ophthalmopathy. J Ophthalmol. 2016;2016:9452687. doi: 1155/2016/9452687. Epub 2016 Jul 17.

18. Romano MR1, Cennamo G2, Breve MA3, Piedepalumbo M3, lovino C3, Velotti N3, et al. Optic nerve compression: the role of the lamina cribrosa and translaminar pressure. Int J Ophthalmol. $2017 \mathrm{Dec}$ 18;10(12):1883-1888. doi: 10.18240/ijo.2017.12.15. eCollection 2017.

19. Blum Meirovitch S1, Leibovitch 11, Kesler A1, Varssano D1, Rosenblatt A1, Neudorfer M1. Retina and Nerve Fiber Layer Thickness in Eyes with Thyroid-Associated Ophthalmopathy. Isr Med Assoc J. 2017 May;19(5):277-2811.

20. Neudorfer M, Blum S, Kesler A, Varssano D, Leibovitch I. Retinal and peripapillary nerve fiber layer thickness in eyes with thyroid-associated ophthalmopathy. Invest Ophthalmol Vis Sci. 2013;54(15):1436

21. Abazari A1, Allam SS, Adamus G, Ghazi NG. Optical coherence tomography findings in autoimmune retinopathy. Am J Ophthalmol. 2012 Apr;153(4):750-6, 756.e1. doi: 10.1016/j.ajo.2011.09.012. Epub 2012 Jan 14.

22. Fernández-Buenaga R, Rebolleda G, Muñoz-Negrete FJ, Contreras I, Casas-Llera P.Macular thickness. Ophthalmology. 2009 Aug;116(8):1587, 1587.e1-3. doi: 10.1016/j.ophtha.2009.02.028.

23. Wijngaarde R, van Lith GH. Pattern EPS in endocrine orbitopathy. Doc Ophthalmol. 1980; 48:327332. doi: $10.1007 /$ BF00141461. 
24. Salvi M1, Spaggiari E, Neri F, Macaluso C, Gardini E, Ferrozzi F, et al. The study of visual evoked potentials in patients with thyroid-associated ophthalmopathy identifies asymptomatic optic nerve involvement. J Clin Endocrinol Metab. 1997 Apr;82(4):1027-30

25. Rutecka-Debniak A, Lubinski W, Krzystolik Z. [Visual evoked potentials in diagnosis and monitoring of optic neuropathy in the course of thyroid ophthalmopathy] Klinika Oczna. 1999; 101:361-365.

26. Pérez-Rico C1, Rodríguez-González N, Arévalo-Serrano J, Blanco R. Evaluation of multifocal visual evoked potentials in patients with Graves' orbitopathy and subclinical optic nerve involvement. Doc Ophthalmol. 2012 Aug;125(1):11-9. doi: 10.1007/s10633-012-9325-2. Epub 2012 May 13.

27. Spadea L1, Bianco G, Dragani T, Balestrazzi E. Early detection of P-VEP and PERG changes in ophthalmic Graves' disease. Graefes Arch Clin Exp Ophthalmol. 1997 Aug;235(8):501-5.

28. Genovesi-Ebert F1, Di Bartolo E, Lepri A, Poggi V, Romani A, Nardi M. Standardized echography, pattern electroretinography and visual-evoked potential and automated perimetry in the early diagnosis of Graves' neuropathy. Ophthalmologica. 1998;212 Suppl 1:101-3.

29. Pawlowski P, Janusz M, Alina BL, Maria G. Pattern electroretinogram (PERG) in the early diagnosis of optic nerve dysfunction in the course of Graves' orbitopathy. Klinika Oczna. 2013; 115:9-12.

\section{Declarations}

- Ethics approval and consent to participate: Written informed consent was obtained from all participants in the study before examination. The tenets of the Declaration of Helsinki were observed, and the study was reviewed and approved by the Ethics Committee of the Faculty of Medicine, Ain Shams University.

- Consent for publication: Not applicable

- Availability of data and materials: The datasets used and/or analysed during the current study are available from the corresponding author

- Competing interests: The authors declare that they have no competing interests regarding the publication of this paper.

- Funding: All authors certify that they have no affiliations with or involvement in any organization or entity with any financial interest (such as honoraria; educational grants; participation in speaker's bureaus; membership, employment, consultancies, stock ownership or other equity interest; and expert testimony or patent-licensing arrangements) or non-financial interest (such as personal or professional relationships, affiliations, knowledge or beliefs) in the subject matter or materials discussed in this manuscript.

- Author Contributions:

- Conceptualization: Amany Abd El-Fattah El-Shazly, Mahmoud Ahmed Elsamkary

- Recruiting required patients and providing their data, in accordance with the desired inclusion criteria: Mahmoud Ahmed Elsamkary 
- Formal analysis: Amany Abd El-Fattah El-Shazly, Mahmoud Ahmed Elsamkary \& Randa Hesham Ali Abdelgawad

- Methodology: Amany Abd El-Fattah El-Shazly, Mahmoud Ahmed Elsamkary, Tamer Abdel Fattah Badran

- Writing - original draft: Amany Abd El-Fattah El-Shazly

- Writing - review \& editing: Amany Abd El-Fattah El-Shazly, Mahmoud Ahmed Elsamkary, Tamer Abdel Fattah Badran \& Randa Hesham Ali Abdelgawad

- Acknowledgements: not applicable

\section{Tables}

Table 1: Demographics in controls and patients with TAO:

\begin{tabular}{|l|c|c|c|c|}
\hline Variable & Control group & Patients with TAO & t-value & p \\
\cline { 2 - 4 } & Mean \pm SD & Mean \pm SD & & \\
\hline Age (years) & $46.63 \pm 13.63$ & $46.35 \pm 13.32$ & 0.09 & 0.09 \\
\hline Unaided VA (LogMAR) & $0.03 \pm 0.07$ & $0.49 \pm 0.19$ & -14.54 & $<0.001$ \\
[Range of Snellen acuities ratios] & {$[20 / 32-20 / 20]$} & {$[20 / 200-20 / 40]$} & & \\
\hline $\begin{array}{l}\text { BCVA (logMAR) } \\
\text { [Range of Snellen acuities ratios] }\end{array}$ & $0.00 \pm 0.00$ & $0.20 \pm 0.24$ & -5.23 & $<0.001$ \\
& {$[20 / 20-20 / 20]$} & {$[20 / 40-20 / 20]$} & & \\
\hline SE (Diopters) & $-0.28 \pm 0.38$ & $-0.29 \pm 0.22$ & 0.18 & 0.86 \\
\hline AL (mm) & $23.16 \pm 0.77$ & $23.01 \pm 0.75$ & 0.89 & 0.37 \\
\hline IOP (mm Hg) & $17.30 \pm 2.88$ & $16.60 \pm 4.74$ & 0.80 & 0.43 \\
\hline CCT (mm) & $540.65 \pm 12.09$ & $537.85 \pm 19.17$ & 0.78 & 0.44 \\
\hline Duration of disease (years) & - & $1.63 \pm 0.70$ & - & - \\
\hline Degree of proptosis & - & $21.25 \pm 3.04$ & - & - \\
\hline Severity score & - & $3.50 \pm 1.13$ & - & - \\
\hline
\end{tabular}

TAO: thyroid-associated ophthalmopathy BCVA: best corrected visual acuity SE: Spherical equivalent
VA: visual acuity

AL: Axial length

Table 2: Optical coherence tomographic measurements of retinal nerve fibre layer (RNFL) thickness in the studied groups: 


\begin{tabular}{|l|c|c|c|c|}
\hline \multirow{2}{*}{$\begin{array}{l}\text { Variable } \\
(\boldsymbol{\mu}) \boldsymbol{)}\end{array}$} & Control group & Patients with TAO & t-value & $\mathbf{p}$ \\
\cline { 2 - 3 } & Mean $\pm \mathrm{SD}$ & Mean $\pm \mathrm{SD}$ & & \\
\hline Average RFNL & $109.4 \pm 1.41$ & $106.66 \pm 2.072$ & 7.02 & $<0.001$ \\
\hline Superior RFNL & $150.18 \pm 1.027$ & $147.78 \pm 0.97$ & 10.74 & $<0.001$ \\
\hline Inferior RFNL & $150.99 \pm 1.29$ & $149.09 \pm 1.87$ & 5.28 & $<0.001$ \\
\hline Temporal RFNL & $79.48 \pm 1.35$ & $78.44 \pm 2.11$ & 2.62 & 0.01 \\
\hline Nasal RFNL & $84.29 \pm 2.17$ & $82.69 \pm 1.74$ & 3.63 & $<0.001$ \\
\hline
\end{tabular}

Table 3: Optical coherence tomographic measurements of macular retinal thickness of the controls and patients with TAO:

\begin{tabular}{|l|c|c|c|c|}
\hline \multirow{2}{*}{$\begin{array}{l}\text { Variable }(\boldsymbol{\mu m}) \\
\text { Central foveal thickness }\end{array}$} & Control group & Patients with TAO & t-value & $\mathbf{p}$ \\
\cline { 2 - 3 } & Mean \pm SD & Mean \pm SD & & \\
\hline SIM & $349.35 \pm 16.97$ & $247.53 \pm 9.63$ & 3.19 & 0.002 \\
\hline IIM & $329.80 \pm 7.46$ & $325.25 \pm 6.26$ & 2.95 & 0.004 \\
\hline TIM & $330.40 \pm 5.38$ & $323.75 \pm 10.08$ & 3.68 & $<0.001$ \\
\hline NIM & $320.20 \pm 8.85$ & $312.10 \pm 12.63$ & 3.32 & 0.001 \\
\hline Mean inner macular ring & $332.49 \pm 6.59$ & $325.48 \pm 5.03$ & 5.35 & $<0.001$ \\
\hline SOM & $298.75 \pm 7.86$ & $293.88 \pm 8.63$ & 2.64 & $<0.001$ \\
\hline IOM & $283.30 \pm 9.81$ & $276.80 \pm 7.38$ & 3.35 & 0.001 \\
\hline TOM & $265.48 \pm 7.57$ & $258.50 \pm 8.96$ & 3.76 & $<0.001$ \\
\hline NOM & $301.03 \pm 21.44$ & $291.98 \pm 13.81$ & 2.24 & 0.03 \\
\hline Mean outer macular ring & $287.16 \pm 5.52$ & $285.88 \pm 5.22$ & 1.07 & 0.29 \\
\hline
\end{tabular}

\begin{tabular}{llll} 
SIM $=$ & Superior of inner macular ring. & SOM $=$ & Superior of outer macular ring. \\
IIM = & Inferior of inner macular ring. & IOM $=$ & Inferior of outer macular ring. \\
\hline $\mathbf{N I M}=$ & Nasal of inner macular ring. & $\mathbf{N O M}=$ & Nasal of outer macular ring. \\
TIM = & Temporal of macular ring. & TOM $=$ & Temporal of outer macular ring.
\end{tabular}

Table 4. Pattern Electroretinogram (PERG) and Pattern visual evoked potential (PVEP) Parameters in the Studied Groups: 


\begin{tabular}{|c|c|c|c|c|}
\hline \multirow[t]{2}{*}{ Variable } & Control group & Patients with TAO & \multirow[t]{2}{*}{ t-value } & \multirow[t]{2}{*}{$\mathrm{p}$} \\
\hline & Mean \pm SD & Mean \pm SD & & \\
\hline \multicolumn{5}{|l|}{ PERG parameters } \\
\hline P50 latency (ms) & $52.68 \pm 0.63$ & $52.72 \pm 0.74$ & -0.26 & 0.80 \\
\hline N95 latency (ms) & $88.93 \pm 6.92$ & $89.51 \pm 4.89$ & -0.44 & 0.66 \\
\hline N35-P50 amplitude $(\mu \mathrm{V})$ & $2.80 \pm 0.57$ & $2.56 \pm 0.27$ & -2.33 & 0.02 \\
\hline P50-N95 amplitude ( $\mu$ V) & $2.73 \pm 0.42$ & $2.82 \pm 0.39$ & -1.05 & 0.30 \\
\hline \multicolumn{5}{|l|}{ PVEP parameters } \\
\hline N75 latency (ms) & $81.43 \pm 6.21$ & $83.01 \pm 4.82$ & -1.27 & 0.21 \\
\hline P100 latency (ms) & $106.16 \pm 2.86$ & $107.34 \pm 3.53$ & -1.64 & 0.11 \\
\hline N75-P100 amplitude ( $\mu$ V) & $12.88 \pm 0.94$ & $13.26 \pm 0.89$ & -1.81 & 0.07 \\
\hline
\end{tabular}

$\mu \mathrm{V}$ : microvolts.

ms: milliseconds

Table 5: Multifocal electroretinographic and statistical findings: five-rings retinal response density (RRD) of the controls and Patients with TAO:

\begin{tabular}{|l|c|c|c|c|}
\hline $\begin{array}{l}\text { Variable } \\
\left(\mathrm{nV} / \mathrm{deg}^{2}\right)\end{array}$ & Control group & Patients with TAO & t-value & $\mathbf{p}$ \\
\cline { 2 - 3 } RRD1 & $48.93 \pm 1.84$ & $46.61 \pm 6.51$ & 2.17 & 0.03 \\
\hline RRD2 & $28.94 \pm 0.95$ & $27.67 \pm 5.14$ & 1.54 & 0.13 \\
\hline RRD3 & $21.30 \pm 1.08$ & $20.75 \pm 1.96$ & 1.55 & 0.13 \\
\hline RRD4 & $17.07 \pm 1.32$ & $16.77 \pm 0.66$ & 1.32 & 0.19 \\
\hline RRD5 & $12.79 \pm 0.93$ & $12.36 \pm 1.003$ & 1.98 & 0.05 \\
\hline
\end{tabular}

RRD: Ring response density

$\mathrm{nV} / \mathrm{deg}^{2}$ : nanovolt per degree square. SD: standard deviation 
Table 6: Multifocal Electroretinographic and Statistical Findings: Five- rings P1 amplitude and latency of the controls and patients with TAO:

\begin{tabular}{|c|c|c|c|c|}
\hline \multirow[t]{2}{*}{ Variable } & Control group & Patients with TAO & \multirow[t]{2}{*}{ t-value } & \multirow[t]{2}{*}{$\mathrm{p}$} \\
\hline & Mean \pm SD & Mean \pm SD & & \\
\hline R1 P1 Amplitude $(\mu \mathrm{V})$ & $0.99 \pm 0.18$ & $0.91 \pm 0.11$ & 2.44 & 0.02 \\
\hline R2 P1 Amplitude $(\mu \mathrm{V})$ & $0.89 \pm 0.02$ & $0.88 \pm 0.05$ & 1.27 & 0.21 \\
\hline R 3 P1 Amplitude ( $\mu$ V) & $0.68 \pm 0.06$ & $0.66 \pm 0.04$ & 1.33 & 0.19 \\
\hline R 4 P1 Amplitude ( $\mu \mathrm{V})$ & $0.63 \pm 0.08$ & $0.60 \pm 0.10$ & 1.64 & 0.10 \\
\hline R 5 P1 Amplitude $(\mu \mathrm{V})$ & $0.59 \pm 0.09$ & $0.56 \pm 0.06$ & 1.40 & 0.17 \\
\hline Ring 1 Latency (ms) & $41.31 \pm 0.59$ & $41.61 \pm 0.87$ & -1.77 & 0.08 \\
\hline Ring 2 Latency (ms) & $41.14 \pm 0.74$ & $41.46 \pm 0.84$ & -1.82 & 0.07 \\
\hline Ring 3 Latency (ms) & $41.54 \pm 0.81$ & $41.73 \pm 1.15$ & -0.85 & 0.40 \\
\hline Ring 4 Latency (ms) & $42.67 \pm 1.03$ & $42.98 \pm 0.92$ & -1.41 & 0.16 \\
\hline Ring 5 Latency (ms) & $43.29 \pm 0.60$ & $43.59 \pm 1.02$ & -1.59 & 0.12 \\
\hline
\end{tabular}

$\mu \mathrm{V}$ : microvolts.

ms: milliseconds

Table 7: Multifocal Electroretinographic and Statistical Findings: Five- rings N1 amplitude and latency of the controls and patients with TAO: 


\begin{tabular}{|c|c|c|c|c|}
\hline \multirow[t]{2}{*}{ Variable } & Control group & Patients with TAO & \multirow[t]{2}{*}{ t-value } & \multirow[t]{2}{*}{$\mathrm{p}$} \\
\hline & Mean \pm SD & Mean \pm SD & & \\
\hline Ring1 N1 Amplitude ( $\mu$ V) & $0.36 \pm 0.02$ & $0.34 \pm 0.01$ & 5.72 & $<0.001$ \\
\hline Ring 2 N1 Amplitude $(\mu \mathrm{V})$ & $0.28 \pm 0.01$ & $0.26 \pm 0.02$ & 4.78 & $<0.001$ \\
\hline $\begin{array}{l}\text { Ring } 3 \text { N1 Amplitude } \\
(\mu \mathrm{V})\end{array}$ & $0.23 \pm 0.02$ & $0.22 \pm 0.01$ & 3.16 & 0.002 \\
\hline Ring 4 N1 Amplitude $(\mu \mathrm{V})$ & $0.19 \pm 0.01$ & $0.18 \pm 0.02$ & 2.11 & 0.04 \\
\hline Ring 5 N1 Amplitude $(\mu \mathrm{V})$ & $0.13 \pm 0.02$ & $0.12 \pm 0.02$ & 2.12 & 0.04 \\
\hline Ring 1 N1 Latency (ms) & $22.88 \pm 1.13$ & $22.73 \pm 0.97$ & 0.64 & 0.53 \\
\hline Ring 2 N1 Latency (ms) & $23.96 \pm 1.23$ & $23.86 \pm 1.03$ & 0.39 & 0.70 \\
\hline Ring 3 N1 Latency (ms) & $24.42 \pm 1.35$ & $24.27 \pm 1.32$ & 0.50 & 0.62 \\
\hline Ring 4 N1 Latency (ms) & $24.32 \pm 1.28$ & $24.59 \pm 0.88$ & 1.12 & 0.27 \\
\hline Ring 5 N1 Latency (ms) & $24.63 \pm 1.62$ & $24.93 \pm 1.12$ & 0.96 & 0.34 \\
\hline
\end{tabular}

$\mu \mathrm{V}$ : microvolts.

ms: milliseconds

Table (8): Multiple regression analysis of different factors affecting CFT in in the patients with TAO: 


\begin{tabular}{|l|c|c|c|c|}
\hline & Beta & B & t & p-level \\
\hline Intercept & & 37.02 & 0.18 & 0.86 \\
\hline Age (years) & -0.22 & -0.16 & -1.78 & 0.09 \\
\hline VA (logMAR) & -0.18 & -9.46 & -0.67 & 0.51 \\
\hline BCVA (logMAR) & -0.28 & -10.06 & -1.22 & 0.24 \\
\hline SE (Diopters) & 0.16 & 7.00 & 1.24 & 0.23 \\
\hline AL (mm) & -0.18 & -2.32 & -1.201 & 0.24 \\
\hline IOP (mm Hg) & -0.06 & -0.13 & -0.36 & 0.72 \\
\hline CCT (mm) & 0.14 & 0.07 & 0.99 & 0.33 \\
\hline Degree of proptosis in mm & -0.56 & -1.76 & -2.29 & 0.03 \\
\hline Duration of (years) & 0.61 & 8.47 & 1.39 & 0.18 \\
\hline RRD 1 (nV/deg $\left.{ }^{2}\right)$ & -0.10 & -0.15 & -0.62 & 0.54 \\
\hline Ring1 P1 Amplitude ( $\mathbf{p V )}$ & 0.13 & 11.57 & 0.92 & 0.37 \\
\hline Ring 1 Latency (ms) & -0.02 & -0.21 & -0.12 & 0.91 \\
\hline Ring1 N1 Amplitude ( $\mathbf{m V )}$ & 0.53 & 422.01 & 1.01 & 0.33 \\
\hline Ring1 N1 Latency (ms) & 0.69 & 6.91 & 1.51 & 0.15 \\
\hline Clinical activity score & -0.62 & -5.27 & -1.09 & 0.29 \\
\hline
\end{tabular}

Regression Summary for Central foveal thickness of active smokers $\mathrm{R}=0.82 \mathrm{R}]=0.67$ Adjusted $\mathrm{R} \square=0.61 \mathrm{~F}(14,85)=12.21 \mathrm{p}$

Table (9): Multiple regression analysis of different factors affecting RNFL thickness in in the patients with TAO:

\begin{tabular}{|l|c|c|c|c|}
\hline & Beta & B & t & p-level \\
\hline Intercept & & 184.24 & 12.06 & $<0.001$ \\
\hline Age (years) & -0.03 & -0.01 & -0.42 & 0.68 \\
\hline VA (logMAR) & -0.03 & -0.37 & -0.29 & 0.77 \\
\hline BCVA (logMAR) & -0.09 & -0.71 & -0.94 & 0.36 \\
\hline SE (Diopters) & 0.02 & 0.17 & 0.27 & 0.79 \\
\hline AL (mm) & 0.10 & 0.27 & 1.48 & 0.15 \\
\hline IOP (mm Hg) & -0.13 & -0.06 & -1.95 & 0.06 \\
\hline CCT (mm) & 0.06 & 0.01 & 0.78 & 0.44 \\
\hline Degree of proptosis & -0.08 & -0.05 & -0.70 & 0.49 \\
\hline Duration of the disease (years) & 0.67 & 2.00 & 3.23 & 0.004 \\
\hline Activity score & -0.81 & -1.48 & -4.53 & $<0.001$ \\
\hline
\end{tabular}


Regression Summary for Central foveal thickness of active smokers $R=0.82 \mathrm{R}]=0.67$ Adjusted $\mathrm{R} \square=0.61 \mathrm{~F}(14,85)=12.21 \mathrm{p}$

\section{Figures}

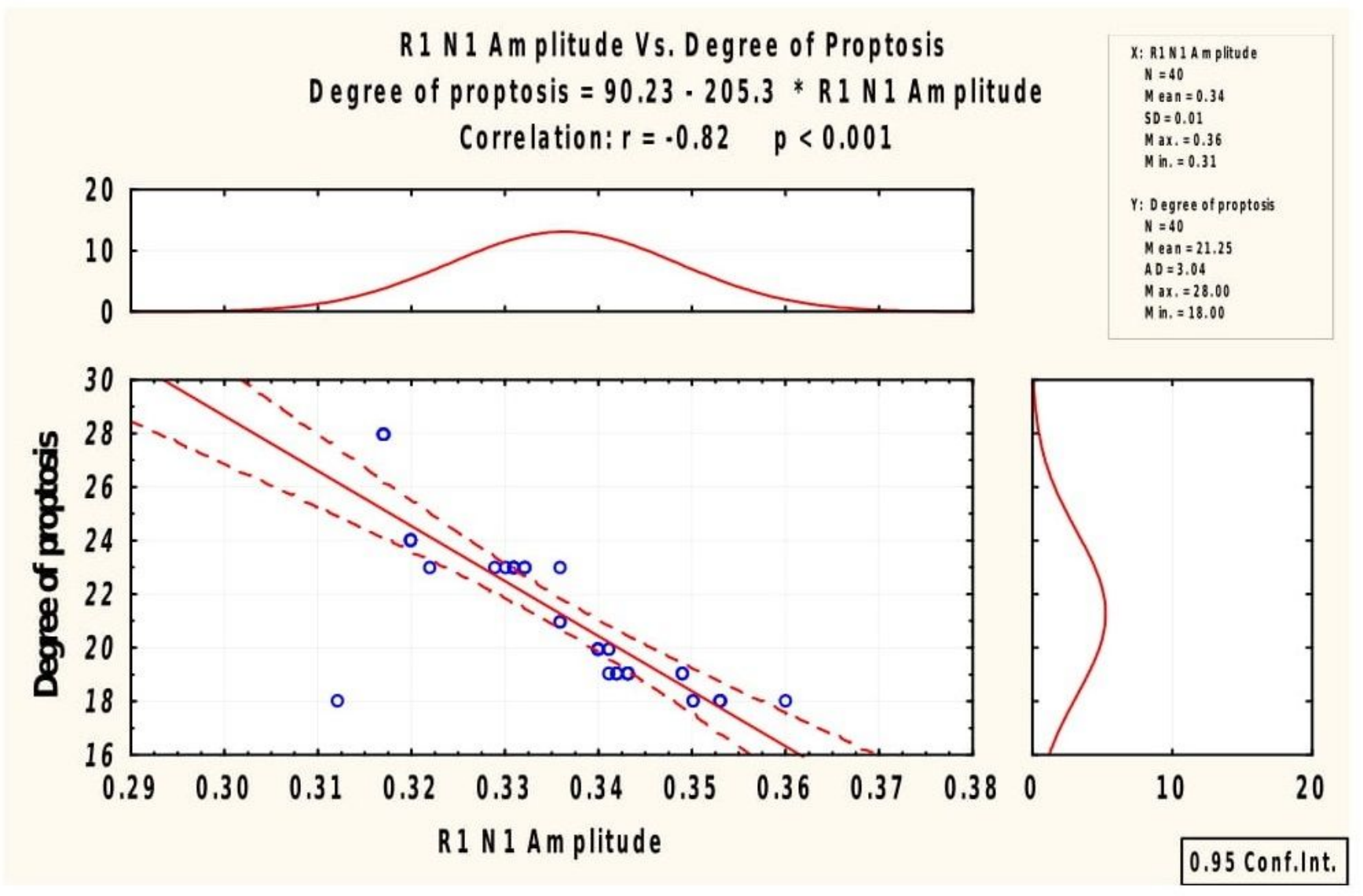

Figure 1

Correlation between R1 N1 amplitude and degree of proptosis. 
R1 1 A m plitude Vs Clinical Activity Score Clinical Activity Score $=33.10 \cdot 88.08 * R 1 N 1$ A m plitude

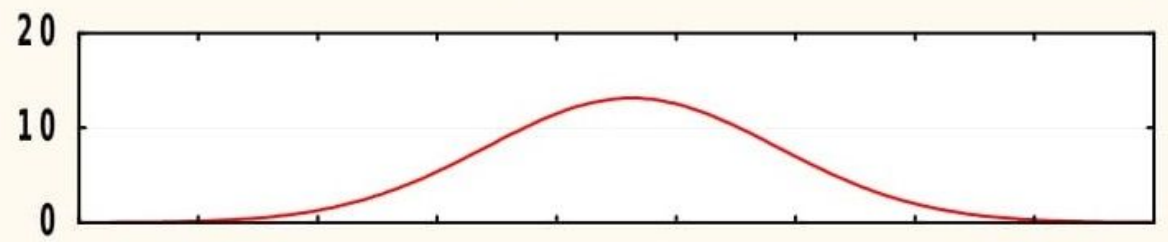

$X: R \perp N 1$ A mplitude $N=40$ $M$ ean $=0.34$ $S D=0.01$

$M$ ax. $=0.36$ $M$ in. $=0.31$

$Y$ : Clinical Activity Score $N=40$

$M$ ean $=3.50$

SD $=1.13$

$M a x_{1}=5.00$

$M$ in. $=2.00$
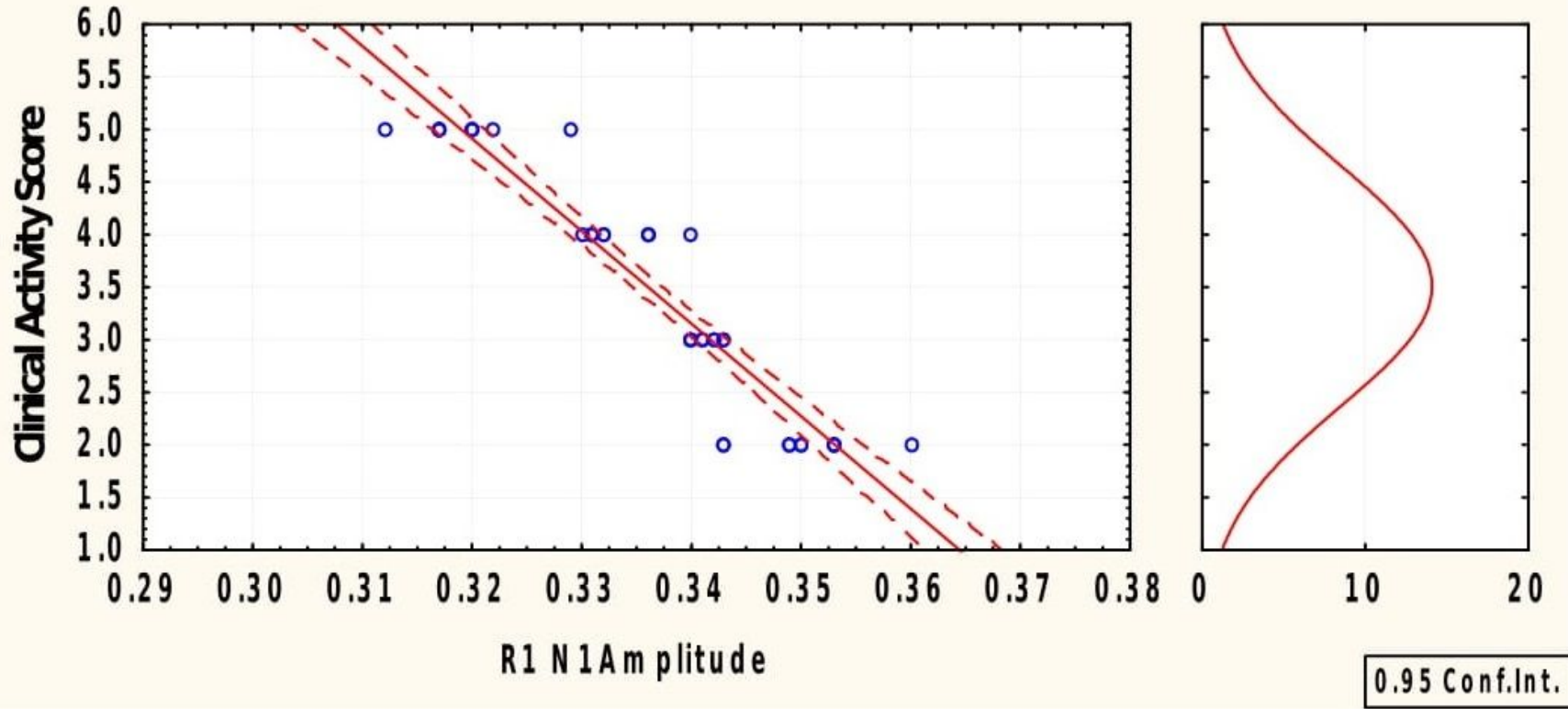

Figure 2

Correlation between R1 N1 amplitude and clinical activity score. 

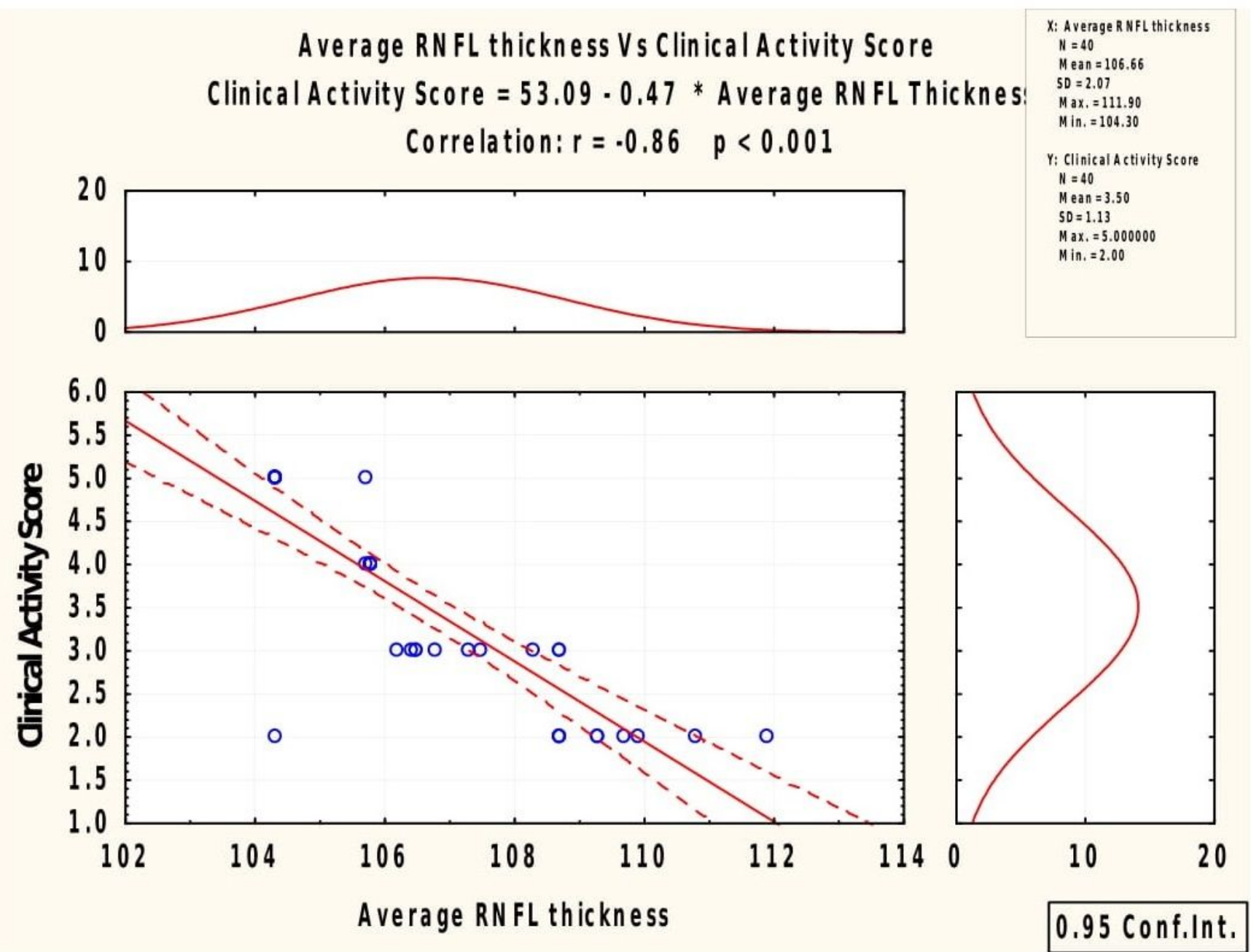

Figure 3

Correlation between Average RNFL thickness and clinical activity score 


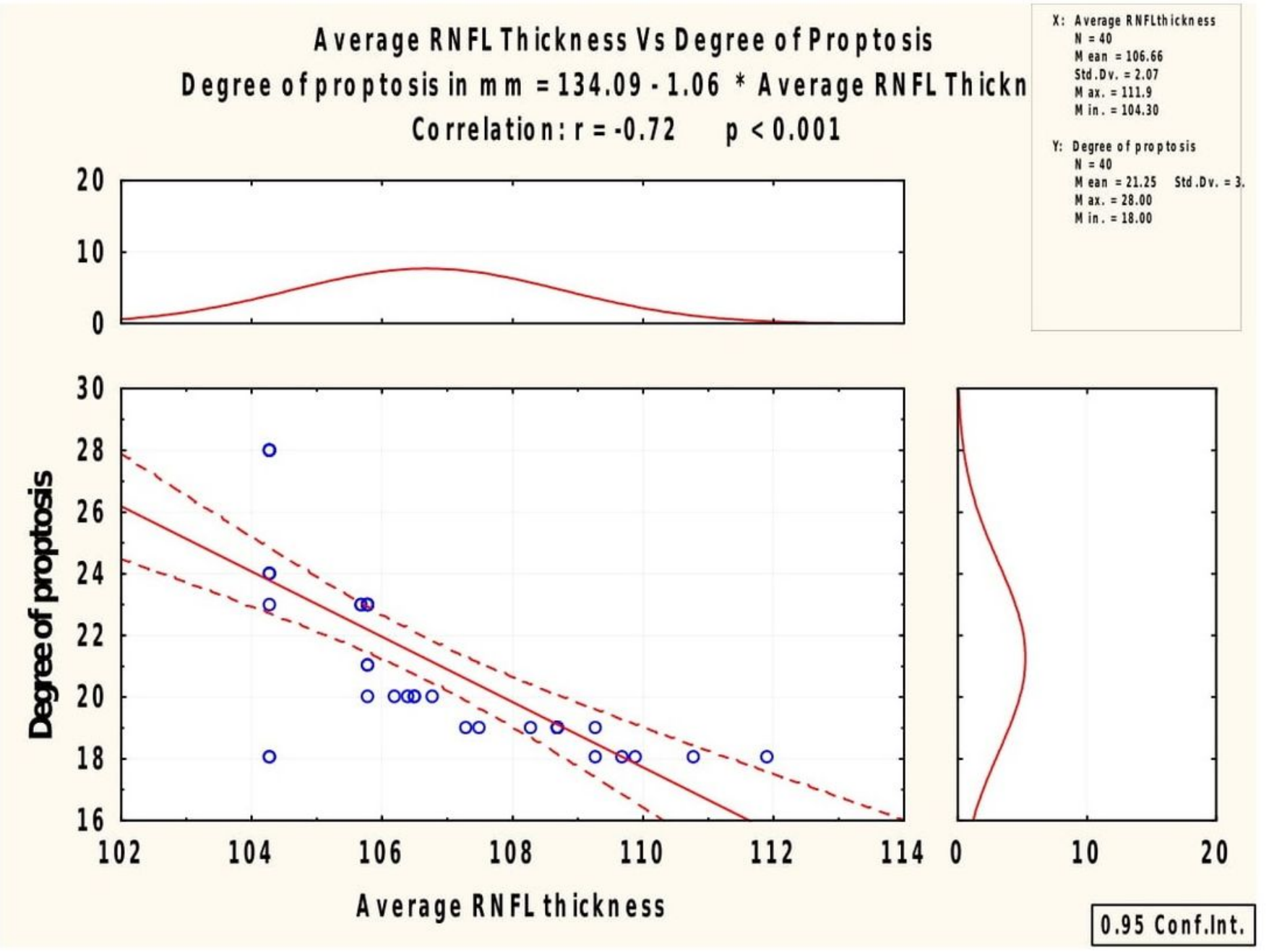

Figure 4

Correlation between Average RNFL thickness and degree of proptosis. 